\title{
Influence of Climate Warming on Arctic Mammals? New Insights from Ancient DNA Studies of the Collared Lemming Dicrostonyx torquatus
}

\author{
Stefan Prost ${ }^{1,2 * a a}$, Nickolay Smirnov ${ }^{3}$, Vadim B. Fedorov ${ }^{4}$, Robert S. Sommer ${ }^{5}$, Mathias Stiller ${ }^{1 \times b}$, Doris \\ Nagel $^{2}$, Michael Knapp ${ }^{1 \mathrm{ac}}$, Michael Hofreiter $^{1 \mathrm{ad}}$
}

1 Research Group Molecular Ecology, Max-Planck Institute for Evolutionary Anthropology, Leipzig, Germany, 2 Department of Paleontology, University of Vienna, Vienna, Austria, $\mathbf{3}$ Institute of Plant and Animal Ecology, Russian Academy of Sciences, Yekaterinburg, Russia, 4 Institute of Arctic Biology, University of Alaska Fairbanks, Fairbanks, Alaska, United States of America, $\mathbf{5}$ Ecology Centre, Christian-Albrechts-University of Kiel, Kiel, Germany

\begin{abstract}
Background: Global temperature increased by approximately half a degree (Celsius) within the last 150 years. Even this moderate warming had major impacts on Earth's ecological and biological systems, especially in the Arctic where the magnitude of abiotic changes even exceeds those in temperate and tropical biomes. Therefore, understanding the biological consequences of climate change on high latitudes is of critical importance for future conservation of the species living in this habitat. The past 25,000 years can be used as a model for such changes, as they were marked by prominent climatic changes that influenced geographical distribution, demographic history and pattern of genetic variation of many extant species. We sequenced ancient and modern DNA of the collared lemming (Dicrostonyx torquatus), which is a key species of the arctic biota, from a single site (Pymva Shor, Northern Pre Urals, Russia) to see if climate warming events after the Last Glacial Maximum had detectable effects on the genetic variation of this arctic rodent species, which is strongly associated with a cold and dry climate.

Results: Using three dimensional network reconstructions we found a dramatic decline in genetic diversity following the LGM. Model-based approaches such as Approximate Bayesian Computation and Markov Chain Monte Carlo based Bayesian inference show that there is evidence for a population decline in the collared lemming following the LGM, with the population size dropping to a minimum during the Greenland Interstadial 1 (Bølling/Allerød) warming phase at 14.5 kyrs BP.

Conclusion: Our results show that previous climate warming events had a strong influence on genetic diversity and population size of collared lemmings. Due to its already severely compromised genetic diversity a similar population reduction as a result of the predicted future climate change could completely abolish the remaining genetic diversity in this population. Local population extinctions of collared lemmings would have severe effects on the arctic ecosystem, as collared lemmings are a key species in the trophic interactions and ecosystem processes in the Arctic.
\end{abstract}

Citation: Prost S, Smirnov N, Fedorov VB, Sommer RS, Stiller M, et al. (2010) Influence of Climate Warming on Arctic Mammals? New Insights from Ancient DNA Studies of the Collared Lemming Dicrostonyx torquatus. PLoS ONE 5(5): e10447. doi:10.1371/journal.pone.0010447

Editor: M. Thomas P. Gilbert, Natural History Museum of Denmark, Denmark

Received January 13, 2010; Accepted April 11, 2010; Published May 27, 2010

Copyright: (c) 2010 Prost et al. This is an open-access article distributed under the terms of the Creative Commons Attribution License, which permits unrestricted use, distribution, and reproduction in any medium, provided the original author and source are credited.

Funding: The authors' work was funded by the Max Planck Society (http://www.mpg.de/). The funders had no role in study design, data collection and analysis, decision to publish, or preparation of the manuscript.

Competing Interests: The authors have declared that no competing interests exist.

* E-mail: stefan.prost@gmi.oeaw.ac.at

a Current address: Gregor Mendel Institute of Molecular Plant Biology, Vienna, Austria

ab Current address: Department of Biology, The Pennsylvania State University, University Park, Pennsylvania, United States of America

ac Current address: Department of Anatomy and Structural Biology, University of Otago, Dunedin, New Zealand

ad Current address: Department of Biology, University of York, York, United Kingdom

\section{Introduction}

Both in public perception and the scientific literature, climate change has become an important topic due to its presumed impact on Earth's ecological and biological systems. Changes in climate such as temperature increase may result in a loss of biodiversity, higher dispersion of diseases, poleward shifts of species ranges, shifts in phenological events (e.g. reproduction, blooming) and even species extinction [1-7]. According to the IPCG the global average temperature is likely to increase by between 1.4 and $5.8^{\circ} \mathrm{C}$ over the period from 1990 to 2100 [8]. Many studies investigated the biological impacts of recent global warming (e.g. [1,2]) concluding that even the moderate warming of the global temperature by half a degree to date already had major effects [2]. The magnitude of abiotic changes in the Arctic is even larger than in temperate and tropical biomes [9-11], further emphasizing the importance of a proper knowledge of the biological effects of climate change, especially in the Arctic [11]. Yet the biological consequences of climate change on high latitudes remain relatively underreported [11] and surprisingly little is known about the effects of climate change on the genetic diversity of species [12], although a proper understanding of the effects of climate change 
on species on the population level is crucial for assessment and prediction of future scenarios.

An excellent approach to improve our knowledge about climate change and its implications is to study the past. The Quaternary period (the past $2.6 \mathrm{My}$ ) was marked by cycles of glacialinterglacial changes $[13,14]$ and thus can be used as a model system to study past demographic changes correlating with climate change. The period following the Last Glacial Maximum (LGM, 25.0-18.0 cal. kyrs BP) was characterized by two major climate warming events: the Greenland Interstadial 1 (Bølling/Allerød warming phase at $14.5 \mathrm{kyrs}$ cal. $\mathrm{BP}$ ) and the transition from the late Pleistocene to the Holocene starting at 11.5 cal. kyrs BP, when a drastic temperature increase occurred within only a few decades [15-17]. These climate warming events resulted in widespread vegetation shifts [18] and faunal extinctions in the Arctic [19].

Ancient DNA provides an outstanding tool to study past population dynamics as it allows a survey of genetic variation in populations over hundreds to thousands of generations and enables the reconstruction of temporal demographic history on a reasonable time scale. In order to reveal biotic responses, reconstructed demographic changes are aligned with the chronology of climatic events inferred from independent lines of evidence. This "phylochronological" approach [12] is a powerful tool to study temporal demographic changes using serial ancient DNA sampling. In one of the first studies, Hadly et al. 2004 [12] analyzed temporal changes of genetic diversity in a single population of the pocket gopher over 2,500 yrs.

The collared lemmings (Dicrostonyx sp.), the northernmost genus of rodents and key species of the arctic community, evolved in dry landscapes of eastern Siberia and were characteristically associated with the dry and cold environment of the tundra steppe during the Pleistocene [20-22]. In order to study the effects of the climate warming events that occurred at $\sim 14.7$ and $\sim 11.5$ cal. kyrs BP on Arctic adapted species, we sampled fossil and modern bones of the collared lemming, Dicrostonyx torquatus, from a single site (Pymva-Shor) in the northern Pre-Urals, for ancient DNA analyses. The layers of this site are dated to 21,910 250 (L6 low), 13,090 \pm 60 (L6 up), 10,000 \pm 250 (L4) yrs BP and modern (surface; calibrated dates are provided in Table S1), thus representing sampling before, during and after climatic events that, based on the ecological preferences of this arctic species should have generated demographic changes.

The paleontological record from the region surrounding and including Pymva Shor shows a decrease in the numbers of fossil remains after the LGM (Figure 4 in [23]), that continues for thousands of years and results in the complete absence of the fossil record for this species around the Holocene thermal optimum (8 to $\sim 4.5$ kyrs).

So far, little is known about species responses to climate change in the Arctic. Our study aims to fill this gap and improve the understanding of past population responses of arctic species to climate change. In the case of the collared lemmings this is particularly important since demographic changes in this species have strong consequences for trophic interactions and ecosystem processes in the Arctic [11,24-30].

\section{Results}

We extracted mitochondrial DNA from 54 ancient and 10 modern collared lemmings from Pymva Shor (Northern Ural, Russia) and 13 ancient samples from Yangana-Pe-4 (Northern Ural, Russia). From all extracts, we successfully amplified and sequenced 282bp of the cytochrome B (CytB) gene and 426bp of the control region $(\mathrm{CR})$. Surprisingly we found the control region to show less variability per nucleotide position than cytochrome $b$. Although this is an unusual result, as the control region usually comprises the most variable part of the mitochondrial genome, this observation is not unprecedented. It is consistent with observations in modern collared lemmings [31] and birds [32], but in contrast to mtDNA variation in other rodents [33].

\section{Inference of temporal demographic changes}

First, we constructed a temporal network, to display the haplotype composition through time (Figure 1). This analysis shows that genetic diversity decreased drastically over time, with the highest amount of genetic variation being present around the LGM and the lowest in the modern population. Nearly all distinct haplotypes (different by more than one substitution from the next nearest haplotype in the network) were lost between 15,200 and $11,500 \mathrm{cal}$. yrs BP. The network also reveals two major haplotypes, which were present in all, or in all but the modern population. The number of haplotypes decreases from ten at 25,200 cal. yrs BP to seven at 15,200 and 11,500 cal. yrs BP and further after 11,500 cal. yrs BP to only three haplotypes in the present (Figure 1 and Table 1).

We tested the possibility that sampling bias resulted in the observed pattern by simulating a temporal data set under a constant population size model. Using average pairwise sequence difference as a summary statistic reflecting the genetic diversity within sample points we found little support for the two youngest sample points (modern and 11,500 cal. yrs BP, Figure S1) under a constant size model with P-values of 0.00988 and 0.00654 respectively, but higher support for the most diverse and oldest sample points $(15,200$ and 25,200 cal. yrs BP, Figure S1) with Pvalues of 0.18757 and 0.22578 respectively. Thus we were able to reject the hypothesis that the low genetic diversity observed at the two youngest data points could be obtained by chance.

Next, we calculated a Bayesian Skyline Plot (BSP) [34] using MCMC based Bayesian inference as implemented in the Software BEAST 1.4.8. [35]. Female effective population sizes at different time points were estimated from the lineage coalescent rate through time. In order to reduce noise introduced by outliers in the data set, we summarized neighboring coalescent intervals into groups and analyzed the data with varying numbers of groups. We analyzed runs with 6,10 and 12 groups. The BSP (Figure 2) shows a decrease in the female effective population size (fNe) starting around the end of the LGM. This fNe decline reaches its maximum between 14,000 and 15,000 cal. yrs BP (assuming a generation time of one year). After a short recovery phase there is another inflection point at around 12,000 cal. yrs BP after which the population size stays approximately constant. However, the highest posterior density (HPD) intervals broaden between 11,500 cal. yrs BP and the present. This population decline followed by a subsequent increase was observed in all analyses independent of the number of coalescence interval groups (i.e 6, 10 or 12). Anyway, the signal magnitude changed, with the strongest signal under a 12 group prior and the weakest signal under a 6 group prior. This result is expected, since fewer groups will cause a strong averaging of population sizes from coalescent intervals preceding and following the bottleneck (data not shown). We further used Tracer 1.4 [36] to compare the results of the BSP reconstruction with a simple constant population model. The calculated $\log 10$ Bayes factor of 1.0 suggests a substantial to strong support for the BSP reconstruction [37].

The mutation rate was estimated at 0.06 (ABC: fragmentation model) and 0.085 (ABC: bottleneck model) to 0.09 mutations/ site/myrs (MCMC method). To confirm that the mutation rate was not only depending on the sample age (i.e if the sequence 


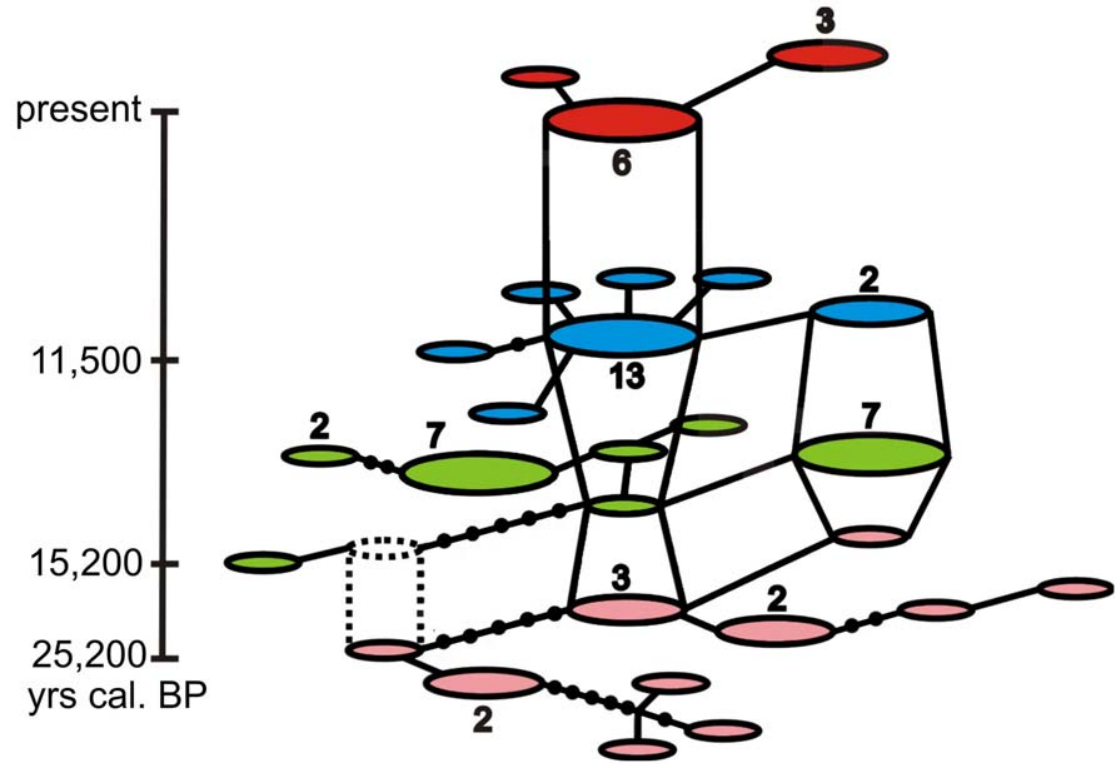

Figure 1. Three dimensional statistical parsimony network. The network is structured in four layers comprising haplotype samplings at different age (rose: 25,200 cal. yrs BP, green: 15,200 cal. yrs BP, blue: 11,500 cal. yrs BP, red: modern). Each circle represents a separate haplotype. The lines between circles show the number of substitutions separating two haplotypes. If the haplotypes are separated by more than one substitution the number of cross lines plus one refers to the actual number of substitutions. Dashed circles and lines indicate an unsampled intermediate haplotype sampled at the previous time-point. Numbers above or below circles indicate the actual number of individuals belonging to this haplotype. Only numbers higher than one are displayed.

doi:10.1371/journal.pone.0010447.g001

data was uninformative) we repeated the BSP analyses with 20 datasets in which sequence data and sample age were randomly shuffled and found that the estimate for our original data set clearly falls outside the range of the estimates from the randomly shuffled datasets indicating that there is sufficient variation in the data to reliably estimate the mutation rate. The marginal densities of the test runs are shown in Figure S2 in the online supplementary material.

Table 1. Statistics summarizing the data.

\begin{tabular}{llllll}
\hline Age (cal. yrs BP) & Seg.Sites & Haplotypes & Pairw. Diff. & Nucleo. Div. & Hapl. Div. \\
\hline Cytochrome B - 282bp & 1 & & & & \\
Modern & 6 & 2 & 0.467 & 0.00165 & 0.467 \\
11.500 & 8 & 6 & 0.689 & 0.00244 & 0.516 \\
15.200 & 12 & 6 & 1.868 & 0.00663 & 0.737 \\
25.200 & & 7 & 3.736 & 0.01325 & 0.901 \\
Control Region - 426bp & 1 & & & \\
Modern & 1 & 2 & 0.200 & 0.00047 & \\
11.500 & 5 & 2 & 0.100 & 0.00023 & 0.200 \\
15.200 & 6 & 4 & 0.679 & 0.00159 & 0.100 \\
25.200 & & 8 & 2.451 & 0.00575 & 0.437 \\
Combined - 708bp & 2 & & & 0.824 \\
Modern & 7 & 3 & 0.667 & 0.00094 & 0.600 \\
11.500 & 13 & 7 & 0.789 & 0.00112 & 0.584 \\
15.200 & 18 & 7 & 2.547 & 0.00360 & 0.774 \\
25.200 & 10 & 6.187 & 0.00874 & 0.945 \\
\hline
\end{tabular}

We estimated different summary statistics for the two genes separately and for the combined region. The number of segregating sites directly reflects the genetic variability and therefore the effective population size. Additionally we provided frequency based statistics such as average pairwise sequence difference. Interestingly we found higher variation in cytochrome $b$ gene than in the control region, which is consistent with observations in modern collared lemmings [31] but in contrast to mtDNA variation in other rodents [33]. (Seg.Sites...segregating sites, Haplotypes...number of haplotypes, Pairw. Diff... average pairwise sequence difference, Nucleo. Div...nucleotide diversity, Hapl.Div...haplotype diversity).

doi:10.1371/journal.pone.0010447.t001 


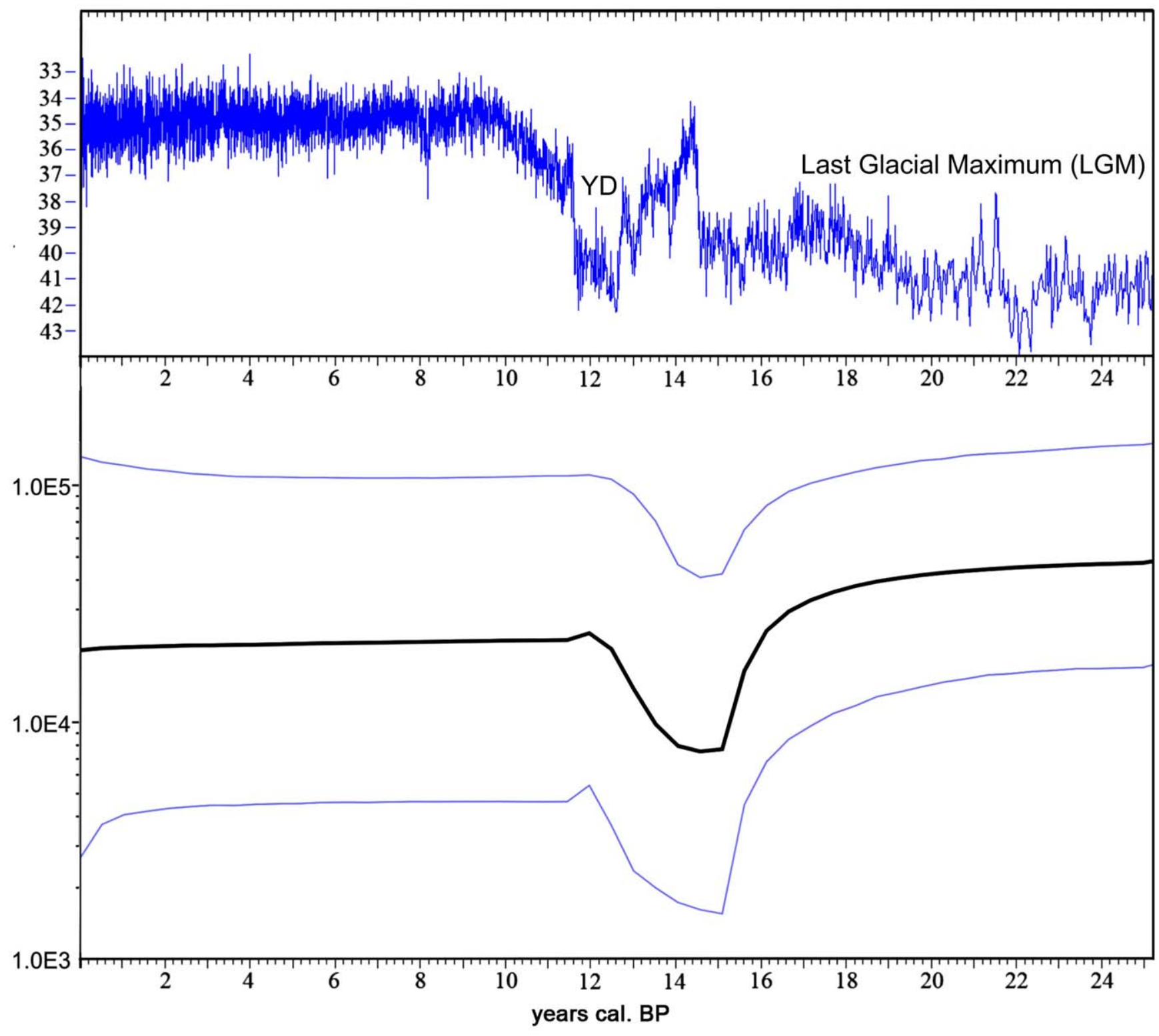

Figure 2. Climate history as derived from the GISP2 ice-core (upper panel) and demographic history of the collared lemming Dicrostonyx torquatus (lower panel) during the last 25,200 yrs. Upper panel: oxygen isotope $\left(\sigma^{18} O\right)$ deviations in \%o ( $y$-axis). The climate graph was calculated using the program CalPal [85]. Lower panel: Bayesian Skyline Plot reconstruction of the temporal demographic history of Dicrostonyx torquatus. The thick black line is the median estimate of the $\mathrm{fNe}$ ( $\mathrm{y}$-axis) over time and the blue lines indicate the $95 \%$ highest posterior density intervals (HPD).

doi:10.1371/journal.pone.0010447.g002

\section{Assessing possible gene flow between locations}

We reconstructed additional statistical parsimony networks including just modern (Figure 3A) and modern and up to 1,000 yrs old samples from Yangana-Pe-4 (Figure 3B) to address the question of whether the data bears signs of modern gene flow and if so to what extend (Figure 3). The modern data shows a restriction of all haplotypes to just a single geographic location, a phylogeographic structure that implies that no recent gene flow occurred between the regions investigated. However, we found the most prominent haplotypes from both PS and Yamal in the 1,000 year old samples from Yangana-Pe-4 (which is situated between Yamal and PS). In total, the samples from Yangana-Pe-4 revealed three haplotypes. Two haplotypes are identical or closely related to the Yamal haplotype (comprising 3 sequences). All other sequences
(10) belong to the major haplotype within PS, indicating historical gene flow from both PS and Yamal into Yangana-Pe-4 or vice versa. Furthermore, four unique, slightly divergent haplotypes (each differing by one mutations from the most common one) were found in the sampling from PS dating to 11,500 cal. yrs BP, which could be interpreted as further evidence of gene flow (see discussion).

In order to verify these results we used the approximate Bayesian computation (ABC) approach [38] to test whether a closed single-population-bottleneck model (no gene flow), an open population-bottleneck model (gene flow following the period of minimum population size) or a constant-population-size model provided the best fit for our data. The posterior probability distribution for the timing of the bottleneck, fNe after and before the bottleneck and the estimated mutation rate of the closed 


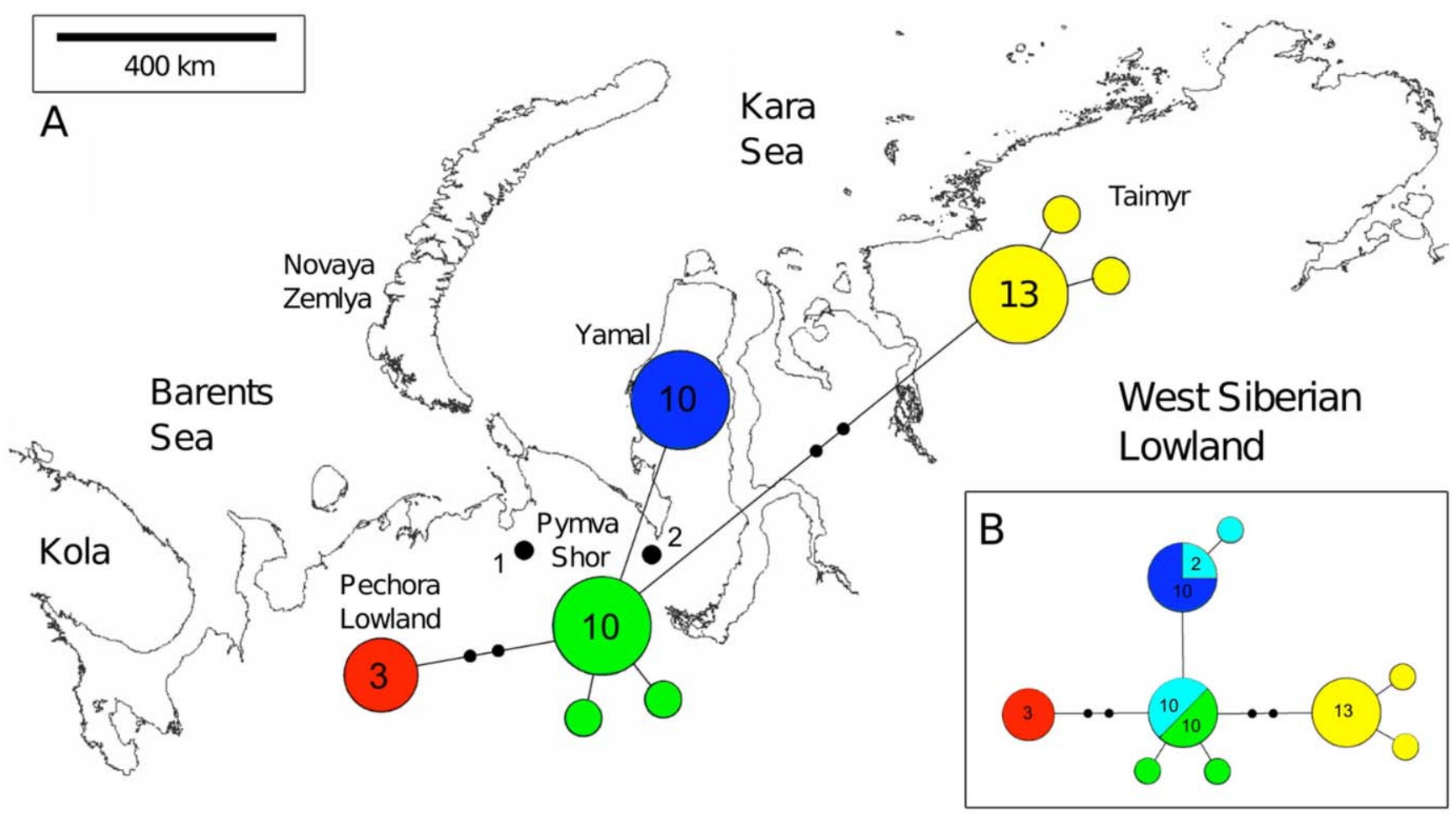

Figure 3. Statistical parsimony networks showing phylogenetic relationships among mtDNA haplotypes from different regions adjacent to Pymva Shor. Main panel: Statistical parsimony network showing phylogenetic relationships among modern mtDNA haplotypes from different regions adjacent to Pymva Shor. Lower right: Statistical parsimony network showing relationships among modern mtDNA haplotypes from different populations adjacent to Pymva Shor including ancient DNA sequences (younger than 1,000 years) from Yangana-Pe-4 (1: Pymva Shor, 2: Yangana-Pe-4). Circles represent haplotypes and colors refer to locality sampled (red: Pechora, green: Pymva Shor, blue: Yamal, yellow: NW-Taimyr and cyan: Yangana-Pe-4). Numbers in the circles refer to the actual number of individuals carrying a haplotype. Only numbers bigger than one are shown.

doi:10.1371/journal.pone.0010447.g003

population-bottleneck model can be seen in Table 2. The posterior probability distributions of both bottleneck models show clear peaks confirming that the genetic data contains sufficient information and that the results are not driven by the priors (Figure 4 for the closed population-bottleneck model or Figure S3 for the open population-bottleneck model). The open populationbottleneck model was supported more strongly $(55.8 \%)$ in the model comparison method applying a rejection and regression step [39] than the closed population bottleneck model (44.2\%) and the constant-population-size model $(0 \%)$, weakly suggesting ancient gene flow between PS and neighboring regions (Figure S4). Histograms of the 6,000 closest estimates can bee seen in Figure S5 showing that the specified models indeed fit the observed data.

Table 2. Parameter - Mode and Quantiles (5\%, 25\%, 95\%).

\begin{tabular}{|c|c|c|c|c|c|c|c|}
\hline \multirow[t]{3}{*}{ Parameter } & \multirow[t]{3}{*}{ Mode } & \multicolumn{6}{|l|}{ Quantile } \\
\hline & & \multicolumn{2}{|l|}{$5 \%$} & \multicolumn{2}{|l|}{$25 \%$} & \multicolumn{2}{|l|}{$95 \%$} \\
\hline & & LLim & ULim & LLim & ULim & LLim & ULim \\
\hline Bottleneck timing & $13,998.5$ & $13,392.9$ & $14,571.4$ & 10785.0 & $16,813.8$ & 146.0 & $40,448.7$ \\
\hline fNe before the $\mathrm{Bn}$ & $28,331.1$ & $25,535.9$ & $31,170.0$ & $14,508.0$ & $42,918.6$ & 269.87 & $17,8071.2$ \\
\hline fNe after the $\mathrm{Bn}$ & $3,690.6$ & $3,591.2$ & $3,785.5$ & $3,117.1$ & $4,114.8$ & 122.9 & $5,053.8$ \\
\hline Modern fNe & $11,090.3$ & $10,221.9$ & $11,969.6$ & $6,589.1$ & 1,5432 & 27.09587 & $46,428.6$ \\
\hline mutation rate & $5.60 \mathrm{e}-05$ & $5.22 \mathrm{e}-05$ & $5.98 \mathrm{e}-05$ & $3.72 \mathrm{e}-05$ & $7.55 e-05$ & $7.09 \mathrm{e}-06$ & 0.000242 \\
\hline Growth rate & $-1.84 \mathrm{e}-04$ & $-1.94 \mathrm{e}-04$ & $-1.72 \mathrm{e}-04$ & $-2.37 \mathrm{e}-04$ & $-1.29 \mathrm{e}-04$ & -0.000753 & 0.000336 \\
\hline fNe scaling factor & 10.2 & 9.2 & 11.5 & 4.33 & 16.4 & 0.08 & 134.2 \\
\hline
\end{tabular}

Mode and quantiles $(5 \%, 25 \%, 95 \%)$ of four model parameters of the ABC analysis. Lower (LLim) and upper (ULim) limits of the respective quantile are provided for the bottleneck timing, the female effective population size after ( $\mathrm{fNe}$ after the $\mathrm{Bn}$ ) and before (fNe before the $\mathrm{Bn}$ ) the bottleneck and the modern effective population size along with the mutation rate estimated (mutations/locus/generation), growth rate and the new fNE scaling factor (to simulate a population reduction).

doi:10.1371/journal.pone.0010447.t002 

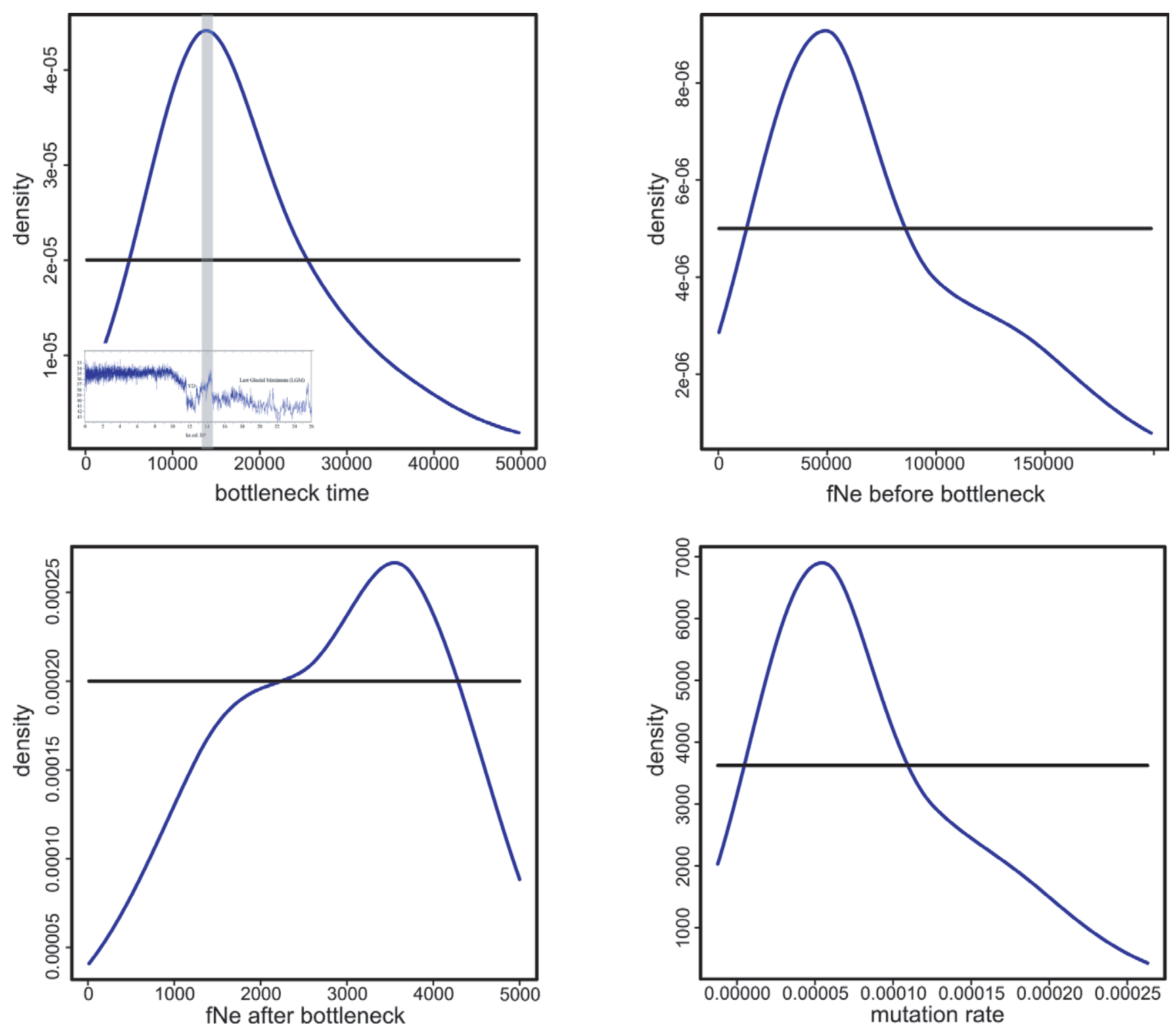

Figure 4. Posterior probability distributions of different model parameters of the ABC analysis. Upper left: Density curve of the bottleneck time. The grey bar shows the $5 \%$ quantile of the highest posterior density, which matches to the first major temperature increase at the Pleistocene-Holocene transition (as indicated in the small climate reconstruction). Upper right: Density curve of the female effective population size prior to the bottleneck. Lower left: Density curve of the female effective population size after the bottleneck. Lower right: Density curve of the mutation rate. The prior distributions are represented as black lines in all the plots.

doi:10.1371/journal.pone.0010447.g004

\section{Discussion}

During the Late Pleistocene the collared lemming, a key species of the arctic community, inhabited large parts of the Holarctic, especially in northern Eurasia [40]. Today, it is present only in the Arctic with a nearly circumpolar distribution (e.g. [40]). It is restricted to dry and treeless tundra and is the only rodent genus that inhabits the polar desert of the northernmost parts of the Eurasian and Canadian Arctic [41,42]. Collared lemmings show a range of unique features that clearly indicate their adaptation to the Arctic environment. In winter they develop a white pelage and enlarged forefeet claws which allow them to dig through compacted snow [43]. Availability of Arctic shrubs and herbs such as those found in dry tundra habitats, are major ecological requirements of collared lemmings $[41,42,44]$. They provide both food and good burrowing conditions. In times of warm and humid climates in the Siberian lowlands, the dry Arctic tundra habitats preferred by collared lemmings shrank and became replaced by southern plant communities [27]. The fossil record shows that during the glacial advances the distribution range of collared lemmings expanded thousands of kilometers to the south while it contracted to the north during warm interglacials $[45,46]$. In addition, to the paleontological record, genetic data from extant populations of collared lemmings in the Eurasian Arctic implies that this species underwent a continent-wide population contraction, possibly during one of the warm interglacials, followed by demographic expansion (e.g. [40,47,48]). Modern DNA sequences of Dicrostonyx torquatus show a restriction of haplotypes to limited geographic regions [40]. Furthermore, within these regional phylogeographical groups, low nucleotide and haplotype diversity 
and star-like haplotype phylogenies were found. These findings suggest regional bottleneck events, probably during the Holocene warming events [40].

Using ancient DNA sequences, we found genetic signs of population decline in the collared lemming in the period following the LGM. The reduction of the effective population size showed its maximum at the Greenland Interstadial 1 (Bølling/Allerød) warming phase at $14,500 \mathrm{yrs} \mathrm{BP}$, which is congruent with predictions based on the ecological preferences of this Arctic species. Thus, the high genetic variation during the LGM most likely reflects a large and stable effective population size during the dry and cold climatic conditions of the Polar Ural and adjacent regions during this time [49], which are optimal for collared lemmings. The temporal network constructed from ancient and modern DNA sequences from a single site (Pymva Shor) in the northern Pre-Urals (Figure 1) shows a marked decline in genetic variation over time. Nearly all distinct haplotypes (differing by more than one substitution from the nearest haplotype in the network) were lost by $11,500 \mathrm{cal}$. yrs BP. Our simulations suggest that given the number of samples analyzed, it is unlikely that the low diversity observed at 11,500 cal. yrs BP and the present was obtained by chance and support the notion of a decline in genetic diversity over time. Such a pronounced demographic reduction is consistent with the existence of a bottleneck during that time. Model-based temporal demographic reconstructions further support our hypothesis of a bottleneck event after the LGM. Both MCMC based Bayesian inference and ABC simulations indicate that the population decline corresponds to the abrupt temperature increase around 14,700 cal. yrs BP during the Greenland Interstadial 1 (Bølling/Allerød) warming event. The fossil record of the region surrounding Pymva Shor showed the highest number of collared lemmings within the coldest and most arid periods $(40,000$ to 45,000 yrs and 21,000 to $18,000 \mathrm{yrs} \mathrm{BP})$ [23]. In layers dating to warmer climatic periods, the proportions of collared lemming fossils decreased. During the Holocene thermal optimum between 8,000 and 4,500 yrs BP fossils of the collared lemmings were absent and these layers are dominated by taiga forest species such as the red vole (most prominent remains) [23]. The decrease in proportion of collared lemming remains along with the temperature increase and the faunal changeover from cold tundra adapted species to taiga species indicate significant effects of climate change on the biota during the Holocene-Pleistocene transition and the Holocene thermal optimum. Furthermore, paleontological and genetic data both indicate a severe population decline in Dicrostonyx torquatus correlating with the abrupt climate change after the LGM. Based on the ecological requirements of the collared lemming and the fossil record one would expect a population decline at 11,500 cal. yrs BP (Holocene-Pleistocene transition) at the beginning of the warm period of the Holocene. However, such a decline was not detectable in the MCMC analysis. Since all divergent haplotypes went extinct before 11,500 generations ago we suggest that most of the power to detect a potential population decline using DNA sequences was lost due to low genetic variation in the modern population. This suggestion is further supported by larger highest posterior density intervals (HPD) in the Bayesian Skyline Plot after 11,500 cal. yrs BP. To overcome this lack of information further sampling between $11,500 \mathrm{cal}$. yrs BP and the present would be necessary in order to obtain a better resolution for the demographic events between these two points in time. In addition given the already small number of haplotypes observed at 11,500 cal. yrs BP, extensive sampling would be necessary to obtain a sufficiently strong signal in the demographic reconstructions. This would be difficult given the low numbers of available samples and the lack of layers between 8,000 and the present in Pymva Shor. It is worthwhile to note, though, that the number of haplotypes detected declines from seven at $11,500 \mathrm{cal}$. yrs BP to only three in the modern population. In combination with the lack of collared lemming fossils during the Holocene climate optimum, these data strongly suggest that a further population size decrease took place after the end of the Pleistocene.

We consider it unlikely that cyclic population density fluctuations that occur at multiple-year intervals generated the observed decline in genetic variation over thousands of years. Population cycles represent more or less regular annual density fluctuations that are reported for small mammals especially voles and lemmings at northern latitudes (e.g. [29,50-54]). Although the causality of these population cycles is under debate, some evidence suggests climate as an important factor [29,54]. Low phases of population cycles represent repeating bottlenecks and are expected to decrease genetic variation due to genetic drift within populations. However, it was suggested that in lemmings, gene flow increases following a population decline and eliminates, or at least reduces the effect of genetic drift on intrapopulation diversity [55-59]. Compiling data on mtDNA polymorphism for 72 lemming populations from 5 species provided no evidence for the hypothesis that a decrease in genetic diversity results from cyclic density fluctuations [55].

Another crucial factor to infer from temporal data is gene flow. The four singleton haplotypes observed at PS at 11,500 cal. yrs BP could indicate possible migration into the sampled population following the strong population decline at approximately 14,500 cal. yrs BP. Although the star-like pattern of haplotypes at 11,500 cal. yrs BP is consistent with a signal of population expansion, with an estimated mutation rate of about 0.09 mutations/site/myrs, 3,000 yrs do not appear to be enough time to accumulate such a number of unique mutations. A potential immigration of individuals from adjacent areas carrying unique haplotypes probably explains the relapse in populations size around 11,500 cal. yrs BP. Analysis of mtDNA variation in modern and recent (up to 1,000 BP) populations on a broad geographic scale revealed a possible contact zone between regional phylogeographic groups at the Yangana-Pe-4 locality but provided no evidence for an effect of ongoing gene flow on genetic diversity in the modern sample from the main sampling locality at Pymva Shor. However, our simulation studies show that there are signs of ancient migration between Pymva Shor and adjacent regions. This finding is consistent with both the MCMC based analysis, which shows a fNe recovery following the bottleneck, and modern data suggesting higher migration rates in collared lemmings following a strong population density reduction [55-59].

Using ancient and modern DNA sampling we were able to show that small mammal demography can be highly dynamic. In the case of the collared lemmings we also found that this species drastically changed its migration pattern over time. Furthermore this study adds to the growing evidence that some populations of modern mammal species from the Arctic are deprived of genetic diversity, a pattern observed for such diverse species as polar bear [60], muskoxen [61] or wolf [62]. We found such a reduction of historical effective population size in an Arctic species to be a direct biotic response to warming climate. Although such a reaction may be expected given the ecological requirements of the collard lemming, it is interesting how exact the signal of climate warming is imprinted in the genetic diversity of this species. The close link between genetic diversity and fossil abundance also shows that the fossil record may indeed reflect species abundance rather than the vagaries of preservation and taphonomy. 
The collared lemming, being adapted to arctic environments will most likely face a further demographic decline and range contraction or even extinction, when the average temperature continues to rise. Such a climate-driven demographic decline and range contraction of the collared lemming, which is a key member of northern communities, would have strong consequences for trophic interactions and ecosystem processes in the Arctic [11,2430]. Since collared lemmings are the main prey for four predators (the snowy owl, the Arctic fox, the long-tailed skua and the stoat), a strong climate-driven population decline in this species would most likely cause a severe reduction in predator populations and may lead to local extinction of the predators themselves [30]. Thus, a proper understanding of the effects and responses of this Arctic key species is crucial for predictions of possible future scenarios in the Arctic biota.

\section{Materials and Methods}

\subsection{Sampling and Radiocarbon Dates}

We sampled 64 Dicrostonyx torquatus remains (left jaws) from Pymva-Shor (PS) in the northern Pre-Urals, for ancient DNA analyses. The layers of this site are dated to 21,910 \pm 250 (L6 up), $13,090 \pm 60$ (L6 low), 10,000 250 (L4) yrs BP and modern (surface). The radiocarbon dates for the different layers were taken from [23], (Table 1). We calibrated the dates using the Fairbanks0107 curve as provided online at http://www.radiocarbon. ldeo.columbia.edu/ and described in Fairbanks et al. 2005 [63]. Radiocarbon dates and calibrated radiocarbon dates are shown in Table S1.We collected 14 left jaws from the lower part of layer 6, 20 from the upper part of layer 6, 20 from layer 4 and 10 from the surface. A further 13 samples were collected from Yangana-Pe-4 (YP4). We included only samples from PS in the temporal demographic reconstructions to avoid bias in the results due to sampling of different populations. Our sampling of 10 to 20 individuals per time-point should provide enough power to detect a demographic change within the sampled period [64]. The samples from YP4 were solely used in additional analyzes to survey for evidence of gene flow.

\subsection{DNA Extraction, Amplification and Sequencing}

Extraction of the 77 left jaws (64 from PS and 13 from YP4) was performed following a silica-based extraction protocol after [65]. We used only left jaws for DNA extraction to rule out the possibility of sampling the same individual twice. About $100 \mathrm{mg}$ to $150 \mathrm{mg}$ of bone powder were used in each extraction, which was carried out in a clean-room environment built for handling ancient DNA to avoid external contamination from modern DNA and PCR products. All 77 extracted lemming remains yielded DNA of sufficient quality to amplify fragments of up to 152bp in length (including primers). Primers for use in the 2-step multiplex approach [66] and the 60 cycle PCR approach [67] (for replication of single missing fragments) were designed using the web-based tool primer3 [68]. Sequences of the primer oligonucleotides are provided in Table S2. Throughout the experiments we monitored for contamination using several controls ( 3 blanks for each extraction of 21 bones and 2 additional PCR blanks per primer pair). Each sequence position was determined from at least two independent amplifications, as misincorporations of nucleotides leading to $\mathrm{C}$ to $\mathrm{T}$ and $\mathrm{G}$ to $\mathrm{A}$ changes are very common in ancient DNA [69-72]. In rare cases of consistent differences between two independent amplifications we performed a third PGR to verify the actual nucleotide sequence. We used the directmultiplex tagging protocol described in [73], but applied it to 2nd step - rather then 1st step amplicons - to create libraries for 454
FLX sequencing. All tagged libraries were quantified using the QPGR method described in [74] and sequenced on a 454 FLX platform (ROCHE). Single fragment amplicons (for missing replicates) were cloned using the Topo TA cloning Kit (Invitrogen, The Netherlands) and sequenced on an ABI 3730 capillary sequencer (Applied Biosystems, USA) [67]. As a result of 454 sequencing errors, we could not determine the exact length of two adenine homopolymer stretches within the 428 bp control region fragment, a well known problem of 454 sequencing $[75,76]$. Therefore, we used the same length for the homopolymer sequence in all modern and ancient DNA sequences.

\subsection{Data Analysis}

The consensus sequences of the 64 samples from Pymva Shor (PS) were then aligned using the alignment editor BioEdit 7.0.8 [77] and transformed into a nexus file using the online converter available at http://www.hiv.lanl.gov/content/sequence/ FORMAT_CONVERSION/form.html. We reconstructed statistical parsimony networks for each time-point (modern, 11,500 cal. yrs, 15,200 cal. yrs and 25,200 cal. yrs BP) separately using the software TCS 1.21 [78]. These networks were then connected to form a three dimensional network by combining haplotypes that were present at different time points. Furthermore, we reconstructed two additional parsimony networks using modern sequences from different regions surrounding PS (Pechora, Yamal and Taimyr, Figure 3A) and sequences from a second paleontological sampling site (Yangana-Pe-4, Figure 3B) to test for possible gene flow. All additional modern sequences were obtained from complete mitochondrial genomes sequenced from specimens from the University of Alaska Museum Mammal Collection, which will be published elsewhere (Fedorov in prep). The sequences used in this study can be accessed via GenBank (Accession numbers: HM008998-HM009023 and HM022324-HM022381), for an overview of the haplotype combinations see Table S3.

In order to test the possibility of sampling effects within our data set we used the software Bayesian Serial Simcoal (BayeSSC) [79] to simulate our temporal data under a constant population size model (fNe ranging from 30,000 to 50,000). We sampled 10 sequences for the modern, 20 for 11,500 cal. yrs BP, 20 for 15,200 cal. yrs BP and 14 for 25,200 cal. yrs BP time-points, respectively. Using average pairwise sequence difference (PD) as summary statistic reflecting the genetic diversity within our data we assessed the probability of sampling a similarly low genetic diversity by chance. We ran the simulation for 450,000 iterations. For each observed PD measurement we calculated its P-value using a onesided test and set the significance level at $\alpha=0.01$. Bayesian Serial Simcoal is a modification of the program Simcoal 1.0 [80] which allows for temporal sampling.

0.3.1 MGMG based Bayesian Inference. To infer demographic history directly from the data we used a Markov Chain Monte Carlo (MCMC) based Bayesian approach as implemented in the software BEAST 1.4.8 [35]. We used the software ModelTest 3.7 [81] to identify the best fitting nucleotide substitution model. The Akaike information criterion (AIC) supported the GTR+I model as best fitting model among those provided by BEAST 1.4.8 [35]. This nucleotide substitution model was used to estimate the genealogy best describing the data. The estimates of the female effective population sizes through time were obtained from the lineage coalescent rate. To smooth the variation of population size estimates over time and make the estimates less susceptible to outliers, several lineage coalescent events were summarized into groups. As the sensitivity of the analyses to pick up population size changes is depending on how many lineage coalescent events are summarized into groups we 
performed separate BEAST runs using 10 groups as default value and we chose group numbers on both sides of this value for our test ( 6 and 12, respectively). For each group number prior, three independent MCMC runs of 20,000,000 iterations each, sampling every 1000th step with a burn-in of 2,000,000 steps were performed. The program TRACER 1.4 [36] was used to verify the effective sample sizes (ESS) and the trace of the MCMC runs as well as to visualize demographic changes through time using a Bayesian Skyline Plot (BSP) [34]. To evaluate the support for the complex BSP prior compared with a simple constant size coalescent prior the Dicrostonyx genealogy was also reconstructed in BEAST 1.4.8 [35] under a constant size tree prior with a uniform prior for the population size (from 0 to 200,000). Posterior probabilities of the genealogies given our data under both population size priors were compared by estimating the $\log 10$ Bayes factor using TRACER 1.4 [36]. Furthermore we repeated the analyses under the BSP tree prior for 20 datasets with randomly shuffled sample ages to test if the estimated mutation rate does not solely depend on the age of the sample points.

0.3.2 Approximate Bayesian Computation. We used the Approximate Bayesian Computation (ABC) approach [38] to test a number of population scenarios. The ABC analysis approximates the posterior distribution by using information from a prior distribution and extensive simulations rather than calculating the likelihood of the data directly. We used the software Bayesian Serial Simcoal (BayeSSC) [79] to simulate temporal data under a closed population-bottleneck model and an open population-bottleneck model including ancient migration. In order to compare both bottleneck models, prior knowledge of the bottleneck timing is crucial to distinguish models that do and do not implement ancient gene flow. We therefore carried out the ABC analysis of the closed single-population-bottleneck first. We then used the estimated bottleneck timing as a prior estimate in the open population-bottleneck model to infer the degree of historical gene flow that best fits the data. R 2.8.1 was then used to perform the rejection algorithm and the local linear regression adjustment and smooth weighting after Beaumont et al. 2002 [38]. In addition, we simulated our data under a single-constantpopulation size model to test whether the null hypothesis of a constant $\mathrm{fNe}$ over time can be rejected. We used uniform priors for the modern fNe, growth rate, event timing and severity, mutation rate and $\mathrm{fNe}$ before and after the bottleneck. Modern $\mathrm{fNe}$ ranges from 1 to 50,000 individuals and the new scaling factor for $\mathrm{fNe}$ after the bottleneck was specified as the fNe from 1 to 200,000 divided by $\mathrm{fNe}$ from 1 to 5,000 (fNe before and after the bottleneck, respectively). We simulated the data under possible values of the mutation rate ranging from 0.01 to 0.2 mutations/ site/myrs and assumed an average generation time of one year for Dicrostonyx torquatus [44]. The software Treepuzzle 5.2 [82] was used to investigate the shape parameter of the gamma distribution of mutation rates (0.02 and 8). Since we merged sequences from $\mathrm{CytB}$ and $\mathrm{GR}$ we incorporated a Kimura 2-Parameter model to allow for heterogeneous mutation rate. Four sample groups were used to summarize the data. The same range of prior values for implied parameters was used in the constant size model. For the population fragmentation model (open population-bottleneck model) we simulated a split between 11,000 and 15,000 generations back in time into 4 populations. We used uniform priors for the modern $\mathrm{fNe}$ between 1 and 20,000. In order to simulate ancient migration, we specified a migration matrix with higher bidirectional migration between each adjacent region and PS and less direct migration between adjacent regions. For the higher migration we assumed a uniform prior of between $10^{-6}$ and 1 and for the lower migration between $10^{-8}$ and $10^{-1}$. We assumed that the migration stopped between present and 1,000 generations back in time as there is no sign for recent migration in the modern data. We chose the number of segregating sites, average pairwise sequence difference and nucleotide diversity as summary statistics, because of their ability to unveil different aspects of population history [83].

We performed simulations for all models comprising 2,000,000 iterations. BayeSSC provides a separate output file containing all calculated summary statistics and parameter values. All required summary statistics of the empirical data were estimated using the software DNAsp v5 [84] and can be seen in Table 1. All R functions specific for the $\mathrm{ABC}$ approach are kindly provided by Mark Beaumont on his homepage (http://www.rubic.rdg.ac.uk/ $\sim$ mab/stuff/). We first applied the rejection algorithm to test the three models against each other as described in [39]. Local linear regression and smooth weighting was then applied using the function calmod(). This function estimates the posterior probability of a particular model using categorical regression.

In the next step we used rejection, local linear regression and smooth weighting to estimate the posterior probability distributions of the demographic parameters of the bottleneck model and the fragmentation model using the locfit function. We applied a tolerance of $p_{\delta}=0.001$ for parameter estimation and the model comparison approach, accepting the 5,000 and 6,000 closest estimates, respectively.

\section{Supporting Information}

Figure S1 Parameter distribution of the average pairwise sequence differences (PD) at the differences. Black arrows represent the empirical estimates. Using P-values we show that low diversity within the modern and the 11,500 cal. yrs BP sampling points are unlikely to be an effect of biased sampling $(\mathrm{P}$ values: Modern...0.00988; 11,500 cal. yrs BP... 0.00654; 15,200 cal. yrs BP... 0.18757 and 25,200 cal. yrs BP ... 0.22578).

Found at: doi:10.1371/journal.pone.0010447.s001 (0.36 MB EPS)

Figure S2 Marginal densities of the mtDNA substitution rate for the analysis using randomly shuffled dates. The distributions centered to the left close to a substitution rate of zero show the results for the randomly shuffled dates. The rate estimated for the original data set is given by the grey distribution with a mean of 9.6E-8.

Found at: doi:10.1371/journal.pone.0010447.s002 (0.73 MB EPS)

Figure S3 Posterior probability distributions of different model parameters in the open population-bottleneck model. Density curves of the female population size for the four modern populations (Ne Population 1-4), the mutation rate (mutation rate), timing of the migration stop (migration stop) and the population split (split timing), the two migration rates between adjacent regions surrounding pymva shor (migration rate 1) and between less direct migration only between adjacent regions (migration rate 2). The $\mathrm{x}$-axes are labeled according to the parameters they refer to.

Found at: doi:10.1371/journal.pone.0010447.s003 (0.44 MB EPS)

Figure S4 Results of the model comparison approach using the rejection - and the regression algorithm after [39]. The open population-bottleneck model allowing for ancient migration $(55.8 \%)$ and the closed population-bottleneck model $(44.2 \%)$ are much stronger supported than the constant population size model $(0 \%)$, thus, favoring a demographic reduction over a constant population size through time. A: Rejection Step, B: Regression Step.

Found at: doi:10.1371/journal.pone.0010447.s004 (0.10 MB EPS) 
Figure S5 Histograms of the summary statistics for the closed population-bottleneck model (A) and the open populationbottleneck model (B). Histograms of the eight summary statistics (Segsites...Segregating Sites and NucDiv...Nucleotide Diversity) used in the model comparison approach after [39] for the closed population-bottleneck model (A) and the open populationbottleneck model (B) to show the fit of the simulated data to the observed values. The green dotted-bashed line represent the empirical values.

Found at: doi:10.1371/journal.pone.0010447.s005 (0.76 MB EPS)

Table S1 Sampling. All samples were taken from two sample sites (Pymva Shor and Yangana-Pe-4) in the Northern Ural, Russia. Radiocarbon dates and layer assignment according to [23] are shown as well as calibrated radiocarbon dates using the Fairbanks0107 curve [63].

Found at: doi:10.1371/journal.pone.0010447.s006 (0.02 MB PDF)

Table S2 Primer-Oligonucleotide Sequences. Primers used in the 2-step multiplex approach [66] and the 60 cycle PCR approach [67].

Found at: doi:10.1371/journal.pone.0010447.s007 (0.04 MB PDF)

Table S3 Haplotype composition of the Dicrostonyx torquatus samples used in the study. The first column shows the combination of the different haplotypes of the cytochrome $\mathrm{B}$ gene $(\mathrm{C})$ and the

\section{References}

1. Parmesan C, Yohe G (2003) A globally coherent fingerprint of climate change impacts across natural systems. Nature 421: 37-42.

2. Root TL, Price JT, Hall KR, Schneider SH, Rosenzweig C, et al. (2003) Fingerprints of global warming on wild animals and plants. Nature 421: 57-60.

3. Thomas CD, Cameron A, Green RE, Bakkenes M, Beaumont LJ, et al. (2004) Extinction risk from climate change. Nature 427: 145-148.

4. Høye TT, Post E, Meltofte H, Schmidt NM, Forchhammer MC (2007) Rapid advancement of spring in the High Arctic. Curr Biol 17: 449-451.

5. Patz JA, Campbell-Lendrum D, Holloway T, Foley JA (2005) Impact of regional climate change on human health. Nature 438: 310-317.

6. Thuiller W, Lavorel S, Araújo MB, Sykes MT, Prentice IC (2005) Climate change threats to plant diversity in Europe. P Natl Acad Sci USA 102: 8245-8250.

7. Sommer RS, Zachos FE (2009) Fossil evidence and phylogeography of temperate species: 'glacial refugia' and post-glacial recolonization. J Biogeogr 36: 2013-2020.

8. Houghton JT, Ding Y, Griggs DJ, Noguer M, van der Linden PJ, et al. (2001) Climate Change 2001: The Scientific Basis Cambridge University Press.

9. ACIA (2005) Arctic Climate Impact Assessment Cambridge University Press.

10. Trenberth KE, Jones PD, Ambenje P, Bojariu R, Easterling D, et al. (2007) Climate Change 2007: The Physical Science Basis Cambridge University Press.

11. Post E, Forchhammer MC, Bret-Harte MS, Callaghan TV, Christensen TR, et al. (2009) Ecological dynamics across the Arctic associated with recent climate change. Science 325: 1355-1358.

12. Hadly EA, Ramakrishnan U, Chan YL, van Tuinen M, O'Keefe K, et al. (2004) Genetic response to climatic change: insights from ancient DNA and phylochronology. PLoS Biol 2: 1601-1609.

13. Imbrie J, Boyle EA, Clemens SC, Duffy A, Howard WR, et al. (1992) On the structure and origin of major glaciation cycles 1 . linear responses to Milankovitch forcing. Paleoceanography 7: 701-738.

14. Tzedakis PC, Andrieu V, De Beaulieu JL, Crowhurst S, Follieri M, et al. (1997) Comparison of terrestrial and marine records of changing climate of the last 500,000 years. Earth and Planet Sc Lett 150: 171-176.

15. Dansgaard W, White JWC, Johnsen SJ (1989) The abrupt termination of the younger dryas climate event. Nature 339: 532-534.

16. Lehman SJ, Keigwin LD (1992) Sudden changes in North Atlantic circulation during the last deglaciation. Nature 356: 757-762.

17. Dansgaard W, Johnsen SJ, Clausen HB, Dahl-Jensen D, Gundestrup NS, et al. (1993) Evidence for general instability of past climate from a 250-kyr ice-core record. Nature 364: 218-220.

18. De Vernal A, Hillaire-Marcel G (2008) Natural variability of Greenland climate, vegetation, and ice volume during the past million years. Science 320: $1622-1625$.

19. Guthrie RD (2006) New carbon dates link climatic change with human colonization and Pleistocene extinctions. Nature 441: 207-209. control region $(\mathrm{H})$. The second column shows the combined number of the specific haplotype combination, the following until the next to last refer to the time-points from Pymva Shor site and the last one refers to the samples from Yangana-Pe-4.

Found at: doi:10.1371/journal.pone.0010447.s008 (0.04 MB PDF)

\section{Acknowledgments}

We are grateful to Magnus Nordborg, Alexei Drummond, Susan Ptak and Beth Shapiro for helpful discussion concerning the methods used. We thank Barbara Hoeffner, Barbara Hoeber, Madlen Siegemund, Ayinuer Aximu-Petri and Christoph Reiterich for technical support, Anna Goropashnaya for help with generating modern sequences (from adjacent regions) and Link Olson for providing modern tissue samples from University of Alaska Museum Mammal Collection; Roger Mundry, Christian Anderson and Joao Sollari Lopes for advice and help with statistical methods, Yvonne Chan and Ann Horsburgh for helpful comments on the manuscript and Knut Finstermeyer for help with some of the figures. Finally, we would like to thank the anonymous reviewers for important suggestions that improved the manuscript.

\section{Author Contributions}

Conceived and designed the experiments: SP MS DN MK MH. Performed the experiments: SP. Analyzed the data: SP. Contributed reagents/ materials/analysis tools: NS VBF. Wrote the paper: SP NS VBF RS MK MH.

20. Agadjanian AK (1976) The history of collared lemmings in the Pleistocene. Beringia in the Cenozoic Era (VL Kontrimavichus, ed) Russian translation series 28: $379-388$.

21. Zazhigin VS (1976) Early evolutionary stages of collared lemmings (Dicrostonychini, Microtinae, Rodentia) as characteristic representatives of Beringian Subarctic fauna. Beringia in the Cenozoic Era (V L Kontrimavichus, ed). pp 280-288.

22. Kowalski K (1995) Lemmings (Mammalia, Rodentia) as indicators of temperature and humidity in the European Quaternary. Acta Zool Cracov 38: 85-94.

23. Golovachov IB, Smirnov NG (2009) The Late Pleistocene and Holocene rodents of the Pre-Urals subarctic. Quatern Int 201: 37-42.

24. Batzli GO, White RG, MacLean SF, Pitelka FA, Collier BD (1980) The herbivore-based trophic system. StroudsburgPA: An arctic ecosystem: the coastal tundra at Barrow, Alaska Dowden, Hutchinson \& Ross. pp 335-410.

25. Stenseth NC, Ims RA (1993) The biology of lemmings Academic Press London.

26. Sittler B, Gilg O, Berg TB (2000) Low abundance of King eider nests during low lemming years in Northeast Greenland. Arctic 53: 53-60.

27. Callaghan TV, Björn LO, Chernov Y, Chapin T, Christensen TR, et al. (2004) Biodiversity, distributions and adaptations of Arctic species in the context of environmental change. Ambio 33: 404-417.

28. Callaghan TV, Björn LO, Chernov Y, Chapin T, Christensen TR, et al. (2004) Effects on the structure of arctic ecosystems in the short-and long-term perspectives. Ambio 33: 436-447.

29. Kausrud KL, Mysterud A, Steen H, Vik JO, Ostbye E, et al. (2008) Linking climate change to lemming cycles. Nature 456: 93-98.

30. Gilg O, Sittler B, Hanski I (2009) Climate change and cyclic predator-prey population dynamics in the high-Arctic. Glob Change Biol 15: 2634-2652.

31. Fedorov VB, Stenseth NC (2002) Multiple glacial refugia in the North American Arctic: inference from phylogeography of the collared lemming (Dicrostonyx groenlandicus). P Roy Soc B-Biol Sci 269: 2071-2078.

32. Pereira SL, Grau ET, Wajntal A (2004) Molecular architecture and rates of DNA substitutions of the mitochondrial control region of cracid birds. Genome 47: 535-545.

33. Fedorov VB, Stenseth NC (2001) Glacial survival of the Norwegian lemming (lemmus lemmus) in Scandinavia: inference from mitochondrial DNA variation. P Roy Soc B-Biol Sci 268: 809-814.

34. Drummond AJ, Rambaut A, Shapiro B, Pybus OG (2005) Bayesian coalescent inference of past population dynamics from molecular sequences. Mol Biol Evol 22: $1185-1192$.

35. Drummond AJ, Rambaut A (2007) BEAST: bayesian evolutionary analysis by sampling trees. BMC Evol Biol 7: 214.

36. Drummond AJ, Rambaut A Tracer v1.4, available from http://beast.bio.ed.ac. $\mathrm{uk} /$ tracer.

37. Kass RE, Raftery AE (1995) Bayes factors. J Am Stat Assoc 90: 773-795. 
38. Beaumont MA, Zhang W, Balding DJ (2002) Approximate Bayesian Computation in population genetics. Genetics 162: 2025-2035.

39. Beaumont MA (2008) Joint determination of topology, divergence time and immigration in population trees. Simulations, Genetics and Human Prehistory. pp $134-154$.

40. Fedorov VB, Fredga K, Jarrell GH (1999) Mitochondrial DNA variation and the evolutionary history of chromosome races of collared lemmings (Dicrostonyx) in the Eurasian Arctic. J Evolution Biol 12: 134-145.

41. Rodgers AR, Lewis MC (1986) Diet selection in Arctic lemmings (Lemmus sibiricus and Dicrostonyx groenlandicus): demography, home range, and habitat use. Can J Zool 64: 2717-2727.

42. Pitelka FA, Batzli GO (1993) Distribution, abundance and habitat use by lemmings on the north slope of Alaska. In The Biology of Lemmings (eds NC Stenseth and RA Ims). pp 214-236.

43. Guthrie RD (1968) Paleoecology of a late Pleistocene small mammal community from interior Alaska. Arctic 21: 223-244.

44. Ognev S (1967) Mammals of the USSR and adjacent countries, Vol. 6, Rodents Israel Program for Scientific Translations.

45. Markova AK, Smirnov NG, Kozharinov AV, Kazantseva NE, Simakova AN, et al. (1995) Late Pleistocene distribution and diversity of mammals in northern Eurasia. Paleontologia I Evolucia 28-29: 5-143.

46. Faunmap Working Group (1996) Spatial response of mammals to late Quaternary environmental fluctuations. Science 272: 1601-1606.

47. Fedorov VB, Goropashnaya A (1999) The importance of ice ages in diversification of Arctic collared lemmings (Dicrostonyx): evidence from the mitochondrial cytochrome b region. Hereditas 130: 301-307.

48. Fedorov VB (1999) Contrasting mitochondrial DNA diversity estimates in two sympatric genera of Arctic lemmings (Dicrostonyx: Lemmus) indicate different responses to Quaternary environmental fluctuations. P Roy Soc Lond B Bio 266: $621-626$.

49. Hubberten HW, Andreev A, Astakhov VI, Demidov I, Dowdeswell JA, et al. (2004) The periglacial climate and environment in Northern Eurasia during the Last Glaciation. Quaternary Sci Rev 23: 1333-1357.

50. Pitelka FA (1957) Some aspects of population structure in the short-term cycle of the brown lemming in northern alaska. Springs Harb Symp Quant Biol 22: 237-251.

51. Pearson OP (1966) The prey of carnivores during one cycle of mouse abundance. J Anim Ecol 35: 217-233.

52. MacLean SFJ, Fitzgerald BM, Pitelka FA (1974) Population cycles in arctic lemmings: winter reproduction and predation by weasels. Arctic Alpine Res 6: $1-12$.

53. Wilson DJ, Krebs CJ, Sinclair ARE (1999) Limitation of collared lemming populations during a population cycle. Oikos 87: 382-398.

54. Ims RA, Henden JA, Killengreen ST (2008) Collapsing population cycles. Trends Ecol Evol 23: 79-86.

55. Blackburn GS, Wilson DJ, Krebs CJ (1999) Dispersal of juvenile collared lemmings (Dicrostonyx groenlandicus) in a high density population. Can J Zool 76: 2255-2261.

56. Ims RA, Andreassen HP (2000) Spatial synchronization of vole population dynamics by predatory birds. Nature 408: 194-196.

57. Andreassen HP, Ims RA (2001) Dispersial in patchy vole populations: role of patch configuration, density dependence, and demography. Ecology 82: 2911-2926.

58. Lin YTK, Batzli GO (2001) The influence of habitat quality on dispersal demography, and population dynamics. Ecol Monogr 71: 245-275.

59. Ehrich D, Jorde PE (2005) High genetic variability despite high-amplitude population cycles in lemmings. J Mammal 86: 380-385.

60. Lindqvist C, Schuster SC, Sun Y, Talbot SL, Qi J, et al. (2010) Complete mitochondrial genome of a Pleistocene jawbone unveils the origin of polar bear. P Natl Acad Sci USA.

61. MacPhee RDE, Tikhonov AN, Mol D, Greenwood AD (2005) Late Quaternary loss of genetic diversity in muskox (Ovibos). BMC Evol Biol 5: 49.

62. Leonard JA, Vilà C, Fox-Dobbs K, Koch PL, Wayne RK, et al. (2007) Megafaunal extinctions and the disappearance of a specialized wolf ecomorph. Curr Biol 17: 1146-1150.
63. Fairbanks RG, Mortlock RA, Chiu TC, Cao L, Kaplan A, et al. (2005) Radiocarbon calibration curve spanning 0 to 50,000 years $\mathrm{BP}$ based on paired $230 \mathrm{Th} / 234 \mathrm{U} / 238 \mathrm{U}$ and $14 \mathrm{C}$ dates on pristine corals. Quaternary Sci Rev 24: 1781-1796

64. Ramakrishnan U, Hadly EA, Mountain JL (2005) Detecting past population bottlenecks using temporal genetic data. Mol Ecol 14: 2915-2922.

65. Rohland N, Siedel H, Hofreiter M (2009) A rapid column-based ancient DNA extraction method for increased sample throughput. Mol Ecol Resources.

66. Roempler H, Dear PH, Krause J, Meyer M, Rohland N, et al. (2006) Multiplex amplification of ancient DNA. Nat Protoc 1: 720-728.

67. Hofreiter M, Capelli C, Krings M, Waits L, Conard N, et al. (2002) Ancient DNA analyses reveal high mitochondrial DNA sequence diversity and parallel morphological evolution of late Pleistocene cave bears. Mol Biol Evol 19: 1244-1250.

68. Rozen S, Skaletsky H (2000) Primer3 on the WWW for general users and for biologist programmers. Methods in Molecular Biology 132: 365-86.

69. Hofreiter M, Jaenicke V, Serre D, von Haeseler A, Pääbo S (2001) DNA sequences from multiple amplifications reveal artifacts induced by cytosine deamination in ancient DNA. Nucleic Acids Res 29: 4793-4799.

70. Stiller M, Green RE, Ronan M, Simons JF, Du L, et al. (2006) Patterns of nucleotide misincorporations during enzymatic amplification and direct largescale sequencing of ancient DNA. P Natl Acad Sci USA 103: 13578-13584.

71. Brotherton P, Endicott P, Sanchez JJ, Beaumont M, Barnett R, et al. (2007) Novel high-resolution characterization of ancient DNA reveals CU-type base modification events as the sole cause of post mortem miscoding lesions. Nucleic Acids Res 1: 5717-5728.

72. Briggs AW, Stenzel U, Johnson PLF, Green RE, Kelso J, et al. (2007) Patterns of damage in genomic DNA sequences from a Neandertal. P Natl Acad Sci USA 104: 14616-14621.

73. Stiller M, Knapp M, Stenzel U, Hofreiter M, Meyer M (2009) Direct multiplex sequencing (DMPS) - a novel method for targeted high-throughput sequencing of ancient and highly degraded DNA. Genome Res 19: 1843-1848.

74. Meyer M, Stenzel U, Hofreiter M (2008) Parallel tagged sequencing on the 454 platform. Nat Protoc 3: 267-278.

75. Margulies M, Egholm M, Altman WE, Attiya S, Bader JS, et al. (2005) Genome sequencing in open microfabricated high density picoliter reactors. Nature 437 : 376-380.

76. Wicker T, Schlagenhauf E, Graner A, Close TJ, Keller B, et al. (2006) 454 sequencing put to the test using the complex genome of barley. BMC genomics 7: 275-286.

77. Hall TA (1999) BioEdit: a user-friendly biological sequence alignment editor and analysis program for Windows 95/98/NT. Nucl Acid S 44: 211-232.

78. Clement M, Posada D, Crandall KA (2000) TCS: a computer program to estimate gene genealogies. Mol Ecol 9: 1657-1660.

79. Anderson CNK, Ramakrishnan U, Chan YL, Hadly EA (2005) Serial SimCoal: a population genetics model for data from multiple populations and points in time. Bioinformatics 21: 1733-1734.

80. Excoffier L, Novembre J, Schneider S (2000) SIMCOAL: a general coalescent program for the simulation of molecular data in interconnected populations with arbitrary demography. J Hered 91: 506-509.

81. Posada D, Crandall KA (1998) Modeltest: testing the model of DNA substitution. Bioinformatics 14: 817-818.

82. Schmidt HA, Strimmer K, Vingron M, von Haeseler A (2002) TREE-PUZZLE: maximum likelihood phylogenetic analysis using quartets and parallel computing. Bioinformatics 18: 502-504.

83. Ramakrishnan U, Hadly EA (2009) Using phylochronology to reveal cryptic population histories: review and synthesis of 29 ancient DNA studies. Mol Ecol 18: 1310-1330.

84. Rozas J, Sanchez-DelBarrio JC, Messeguer X, Rozas R (2003) DnaSP, DNA polymorphism analyses by the coalescent and other methods. Bioinformatics 19: 2496-2497.

85. Eninger B, Joeris O, Danzeglocke U (2007) CalPal-2007, Cologne radiocarbon calibration and palaeoclimate research. http://www.calpal.de/, downloaded 2009. 